\title{
PHARMACOECONOMIC ANALYSIS OF DASATINIB AS A SECOND-LINE THERAPY IN PATIENTS WITH CHRONIC MYELOGENOUS LEUKEMIA
}

\author{
Kulikov A.U., Protsenko M.V. \\ Pharmacoeconomic Study laboratory, I.M. Sechenov First Moscow State Medical University
}

\begin{abstract}
CML) is among the nosologies included in the governmental program for supply of medicines to patients with hemophilia, mucoviscidosis, pituitary dwarfism, Gaucher disease, malignant neoplasms in lymphoid, hematopoietic and related tissues, multiple sclerosis, and also after organ and (or) tissue transplantation. Only one medicine, imatinib, is currently available to CML patients within this program. However, $20 \%$ to $35 \%$ of CML patients have intolerance or develop resistance to imatinib. Therefore, this patient group should receive treatment with the second-generation tyrosine-kinase inhibitors, in particular dasatinib. Pharmacoeconomic analysis is required for dasatinib to be included in the list of expensive medicinal products. This pharmacoeconomic study investigates the use of dasatinib as a second-line CML therapy, based on "cost-effectiveness» and «budget impact» analysis. The study demonstrated that in terms of cost-effectiveness analysis, dasatinib is a strictly preferred alternative as compared to the high doses of imatinib. Moreover, taking into account consumption rate of dasatinib in the real world settings, it is possible to provide treatment to $100 \%$ of patients with resistance and/or intolerance to imatinib without additional funding above the total federal budget for CML treatment in 2014.
\end{abstract}

Key words: pharmacoeconomics, chronic myelogenous leukemia, dasatinib, imatinib, list of expensive medicinal products, budget impact analysis, costeffectiveness analysis, pharmacoeconomic modeling.

\section{Introduction}

The governmental program of supply of medicines to persons with hemophilia mucoviscidosis, pituitary dwarfism, Gaucher disease, malignant neoplasms in lymphoid, hematopoietic and related tissues, multiple sclerosis, and also persons after organ and (or) tissue transplantation (hereinafter referred to as «high cost nosology program»), which was launched in 2008 [2], made advanced drug therapy available to patients with severe diseases, thus considerably improving the prognosis of their treatment. Over the next several years, up until 2015, the list of medicinal products covered by the federal budget within this program (a list of expensive medicinal products) remained unchanged. And only in 2015 five new medicines, including lenalidomide, anti-inhibitor coagulant complex, moroctocog alfa, coagulation factor VIII + Von Willebrand factor, and velaglucerase alfa, were added to the list. However, additional medicinal products for CML treatment were not included in the list
[3]. Currently, only one medicinal product, imatinib, which belongs to the first-generation tyrosine-kinase inhibitors (TKI), is provided to CML patients in frame of under the high cost nosology program.

The launch of imatinib in the pharmaceutical market in 2001 led to a noticeable improvement in CML treatment efficacy and also to an increase in the five-year survival rate from $27.1 \%$ in $1990-1992$ to $48.7 \%$ in 2002 $2004[1,12]$. However, a considerable share of patients $(25 \%$ to $35 \%$ of all CML patients, based on different estimates) develop resistance and/or have intolerance to imatinib therapy [1]. Therefore, due to these factors imatinib therapy demonstrates an inadequate efficacy level in a significant number of patients, which results in faster disease progression rates and, consequently, in a shorter life expectancy. Furthermore, resistance to imatinib requires dose increase from $400 \mathrm{mg}$ to $600 \mathrm{mg}$ or $800 \mathrm{mg}$ daily, while intolerance to imatinib may lead to the treatment discontinuation.

Second-generation tyrosine-kinase inhibitors (TKI-2), such as dasatinib and nilotinib, were developed to address the problem of the treatment of patients with resistance and/or intolerance to imatinib. Clinical studies demonstrated high efficacy of TKI-2 as a second-line CML therapy. For example, in a registration study of dasatinib in chronic-phase CML patients, six-year survival rate was $71 \%$, with major molecular response rate over six years being $46 \%$ [15].

It is important to emphasize that continued therapy with imatinib in patients with resistance and/or intolerance to imatinib not only decreases treatment efficacy, but also reduces cost effectiveness of the healthcare budget expenditure on CML treatment. It was demonstrated that in 2012-2013 40\% of funds allocated under the high cost nosology program to provide treatment to CML patients were spent with inadequate cost effectiveness [13]. However, the CML treatment optimization process is limited by the fact that currently none of second-generation TKIs is included in the list of expensive medicinal products. Second-generation inhibitors are procured only from regional budgets, and their availability to patients largely depends on the financial capacity of each particular region. Inclusion of the second-generation TKIs in the list of medicinal products supplied from the federal budget under the high cost nosology program is a possible way to address the problem of treatment of CML patients with resistance and/or intolerance to imatinib.

In accordance with Decree of the Government of the Russian Federation No. 871 dated August 30, 2014 On the Approval of the Rules for Drafting Lists 
of Medicinal Products for Human Use and the Minimal Range of Medicinal Products Required to Provide Medical Care [4], for medicinal products to be included in the list of expensive medicinal products, the results of a pharmacoeconomic evaluation, budget impact analysis and cost-effectiveness analysis, should be taken into account. Therefore, the objective of this study was to conduct a pharmacoeconomic analysis of the second-generation TKI, dasatinib, as a second-line therapy in CML patients with resistance and/or intolerance to imatinib.

\section{Materials and methods}

This study was based on the «decision tree» model and included costeffectiveness and budget impact analyses. The study results are presented with reference to the governmental medicine supply program with account of direct TKI drug therapy costs. Treatment costs were estimated based on the registered maximum ex-factory prices for dasatinib and imatinib, as well as weighted average tender price for imatinib during government procurement under the high cost nosology program. A two-year time horizon was used for cost-effectiveness analysis, and a one-year time horizon was used for budget impact analysis, which is consistent with the annual budget planning under the high cost nosology program. The discount rate in cost-effectiveness analysis was set at $3 \%$. It also should be noted that budget impact analysis was conducted for the entire CML patient population, administered both firstline and second-line therapy, since funds for this disease under the high cost nosology program are allocated for the entire patient population [6].

The first stage of this pharmacoeconomic study comprised a retrospective clinical efficacy analysis that included the selection of an appropriate effectiveness criteria and the search for relevant efficacy values for the studied products [8]. The development of second-generation TKIs entailed fundamental changes in the treatment tactics for patients with resistance and/or intolerance to imatinib, provided that such patients can be shifted to the second-line therapy. Costeffectiveness analysis used the results of Phase II clinical study by Kantarjian et al., 2009 [5], which compared the use of dasatinib and high-dose imatinib for chronic-phase CML patients with resistance and/or intolerance to imatinib. The study enrolled 150 patients, with 101 patients administered dasatinib at a dose of $140 \mathrm{mg}$ daily, and 49 patients on imatinib at a dose of $800 \mathrm{mg}$ daily. The clinical study demonstrated that, after two years of therapy, $44 \%$ of patients in the dasatinib group achieved a complete cytogenetic response (CCyR) versus only $18 \%$ of patients in the imatinib group. Since CCyR achievement is a clinically significant predictor of overall and disease-free survival rates in case of CML [14], this clinical endpoint was selected as an effectiveness criteria for the purpose of pharmacoeconomic assessment.

Only cost of the drug therapy with TKIs was considered. In addition, based on the current national guidelines on CML treatment and relevant prescribing information, the pharmacoeconomic study used the following dosing regimens for the medicinal products of interest $[1,9]$. Imatinib as a first-line therapy - $400 \mathrm{mg}$ daily for chronic-phase CML patients and $800 \mathrm{mg}$ daily for accelerated/blast-phase CML patients and also for chronic-phase CML patients where the standard dose was ineffective. Therefore, an assumption was made for modeling that all patients with CML in advanced phases and chronic-phase CML patient with ineffective standard dose were administered imatinib at the maximum daily dose of $800 \mathrm{mg}$. Dasatinib as a second-line therapy is administered at a dose of $100 \mathrm{mg}$ daily in chronic-phase CML or $140 \mathrm{mg}$ daily in accelerated/blast-phase CML.

At the first stage of analysis, annual treatment cost per one CML patient was estimated for imatinib (standard and elevated doses) and dasatinib (as a second-line therapy), based on their registered maximum ex-factory prices. The use of the registered maximum ex-factory prices was justified by the fact that no other comparable price levels were available for imatinib and dasatinib. Dasatinib cost analysis used the minimum ex-factory price for the original medicinal product Sprycel ${ }^{\circledR}$, while imatinib cost analysis included ex-factory prices for both the original medicinal product Glivec ${ }^{\circledR}$ and available generics (Imatinib $^{\circledR}$, Imatinib-TL ${ }^{\circledR}$, Imagliv ${ }^{\circledR}$, Imatib ${ }^{\circledR}$, Gistamel ${ }^{\circledR}$, Philachromin-FS ${ }^{\circledR}$, Imatinib-Teva ${ }^{\circledR}$, Neopax $^{\circledR}$, Genfatinib $^{\circledR}$ ) [9].

Weighted average maximum ex-factory price for imatinib calculated based on the actual shares of various trade names in government tenders under the high cost nosology program in 2014: the share of Glivec $^{\circledR}$ was $17.59 \%$, Genfatinib ${ }^{\circledast}-30.77 \%$, Philachromin- $\mathrm{FS}^{\circledR}-41.84 \%$, Neopax ${ }^{\circledast}$ $4.65 \%$, Imatib ${ }^{\circledR}-2.72 \%$, Gistame ${ }^{\circledR}-1.59 \%$, Imatinib-Teva ${ }^{\circledR}-0.84 \%$.

Estimated annual treatment cost is provided in figure 1 . The cost of annual treatment with imatinib, based on the registered maximum ex-factory prices, varies depending on the trade name from RUB 330,106 to RUB $1,060,301$ per one chronic-phase CML patient in the first-line therapy; and from RUB 660,212 to RUB 2,120,601 per one accelerated/blast-phase CML patient in the first-line therapy and also for patients administered elevated doses of imatinib, irrespectively of CML phase. The cost of annual treatment with dasatinib amounts to RUB 1,720,111 for chronic-phase CML and RUB $1,722,263$ for accelerated/blast-phase CML in the second line of therapy per one patient.

In addition, «budget impact» analysis took into account the real cost of annual treatment with imatinib, which was estimated based on the weighted average tender price of imatinib in frame of the high cost nosology program in 2014. The weighted average tender price for imatinib, independent of the trade name, amounted to RUB 2.17 per $1 \mathrm{mg}$ [10], with the cost of annual treatment per one patient amounting to RUB 316,820 for chronic-phase CML and RUB 633,640 in other cases.

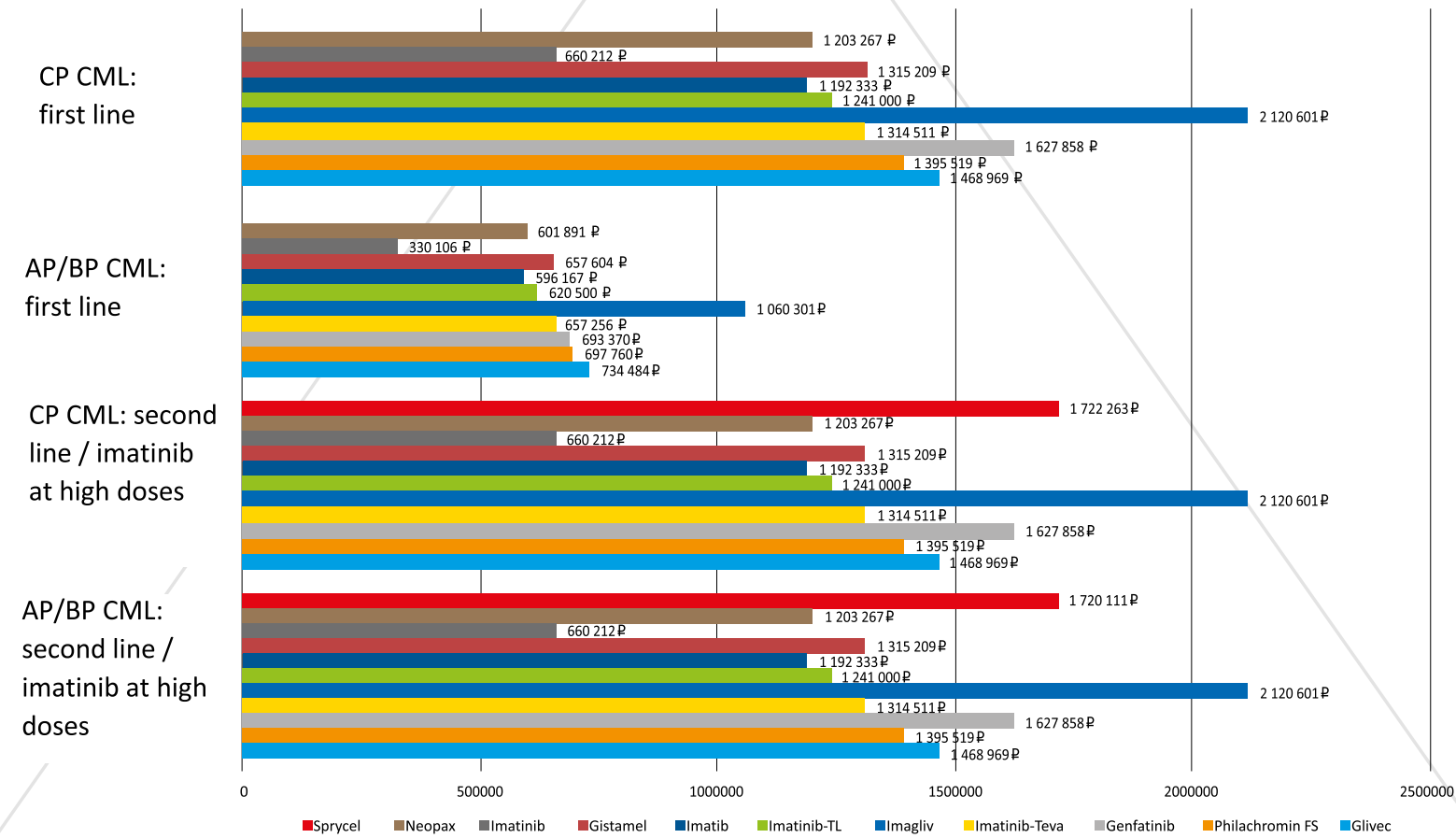

Figure 1. Cost of annual CML treatment with imatinib and dasatinib per one patient (based on the registered maximum ex-factory prices). CP - chronic phase, AP/BP - accelerated phase or blast phase.

*While at the time of this study, daily dose of dasatinib for chronic-phase CML patients was $140 \mathrm{mg}$ daily according to the prescribing information, the current approved daily dose is $100 \mathrm{mg}$ daily. 


\section{Results}

\section{Cost-effectiveness analysis}

Cost-effectiveness analysis compared the costs of one effectiveness unit for dasatinib and high-dose imatinib in second-line therapy, in this way enabling to choose the product that provides maximum clinical effect within the same budget. As an effectiveness criteria, this study used the share of chronic-phase CML patient who achieved CCyR after two years treatment with dasatinib or imatinib at high doses. Analysis was carried out using the registered maximum ex-factory prices for imatinib and dasatinib. Average cost-effectiveness ratio for imatinib at high doses, with account of all trade names of the medicinal product, was RUB 145,925 for achievement of CCyR by $1 \%$ of patients after two years of therapy. The corresponding cost-effectiveness ratio for dasatinib was RUB 75,936 (fig. 2).

One-way sensitivity analysis based on dasatinib price parameter showed that, when dasatinib price is increased by $25 \%$, the cost-effectiveness ratio for dasatinib still remains lower than that for imatinib and amounts to RUB 94,920 . Therefore, in terms of cost-effectiveness analysis dasatinib is a dominant treatment alternative versus imatinib at high doses, when used in patients with resistance to imatinib [7].

\section{Budget impact analysis}

Budget impact analysis was carried out for a population of 7,100 CML patients, with $25 \%$ of them being resistant and/or having intolerance to imatinib therapy. An overwhelming majority of patients have chronic-phase CML - 93\%, with the other patients being in the acceleration and blast phase [16]. During modeling, several scenarios for budget impact analysis were considered, which took into account different price levels for the medicinal products and different penetration rate of dasatinib (share of imatinib-resistant patients administered dasatinib as a second-line therapy). The main and final stage of budget impact analysis was to compare the estimated budget for CML treatment with dasatinib as a second-line therapy with overall cost of treatment of CML patients in Russia in 2014 (RUB 4.53 billion, including funds allocated from the federal budget under the high cost nosology program and funds allocated from regional budgets).

First of all, calculations were made based on the registered maximum ex-factory prices for imatinib and dasatinib. When imatinib is used, whether as a first-line or a second-line CML therapy, the estimated budget (base case scenario) for the treatment of the total CML patient population account to RUB $6,583,418,231$ annually. The use of dasatinib as a second-line therapy in $100 \%$ of imatinib-resistant patients required additional costs of RUB 455 million. However, budget increase did not exceed $7 \%$, with the new budget amounting to RUB $7,039,160,202$ annually. When dasatinib was administered only to $50 \%$ of patients who required a switch from the first-line to the second-line therapy, the treatment budget for all CML patients would amount to RUB $6,811,289,127$ annually, exceeding the base case scenario by $3.5 \%$. Additionally, a possibility to optimize the CML treatment budget by procuring the cheapest trade name of imatinib (Imatinib $\otimes$, Biocad CJSC, Russia) was considered. Optimized imatinib procurement, when dasatinib is used as a second-line therapy in $100 \%$ of relevant patients, will reduce the base case scenario by $25 \%$ to RUB 4,934,326,158.

At the final stage of budget impact analysis, the estimated budget (based on the tender price for imatinib and the registered maximum ex-factory price for dasatinib) was compared against the actual total budget for CML treatment in Russia in 2014 (fig. 3). The modeling process took into account patients adherence to treatment in the real world settings.

Based on retrospective analysis by Esposti et al. 2014 [11], it was suggested that $86.4 \%$ of patients demonstrated maximum $100 \%$ compliance rate and took dasatinib strictly in accordance with the recommended regimen, with the remaining $13.6 \%$ of patient demonstrating $80 \%$ compliance rate. Thus, when dasatinib is prescribed to $100 \%$ of imatinib-resistant patients, the estimated annual CML budget would amount to RUB 4,775,571,612, which is RUB 245 million above the actual CML budget in 2014. On the other hand, $82 \%$ of patients who need a second-line CML therapy can be switched to dasatinib without additional funding.

Moreover, the actual dasatinib consumption within the drug supply program (in frame of the regional budgets) in 2014 was analyzed. This analysis demonstrated that the actual annual dasatinib consumption per one patient was 9.01 packages of the medicinal product instead of 12.6 packages in case of $100 \%$ compliance rate [10]. Low dasatinib consumption rate could be explained by low compliance levels among patients, temporary discontinuation of dasatinib in case of side effects of the 3-4 severity grades, and also by the fact that patients started dasatinib therapy at different time points from the beginning of the calendar year. Taking into account the corercponding factor of actual dasatinib consumption, the expected annual CML budget, when dasatinib is administered to $100 \%$ of imatinib-resistant patients, amounted to RUB $4,067,637,875$. Therefore centralized dasatinib procurement under the high cost nosology program will save RUB 463 million or $10 \%$ of the actual CML budget in 2014 .

\section{CER - the cost of achieving a complete \\ cytogenetic response in the second year of therapy by $1 \%$ of the patients in the second line in the chronic phase}

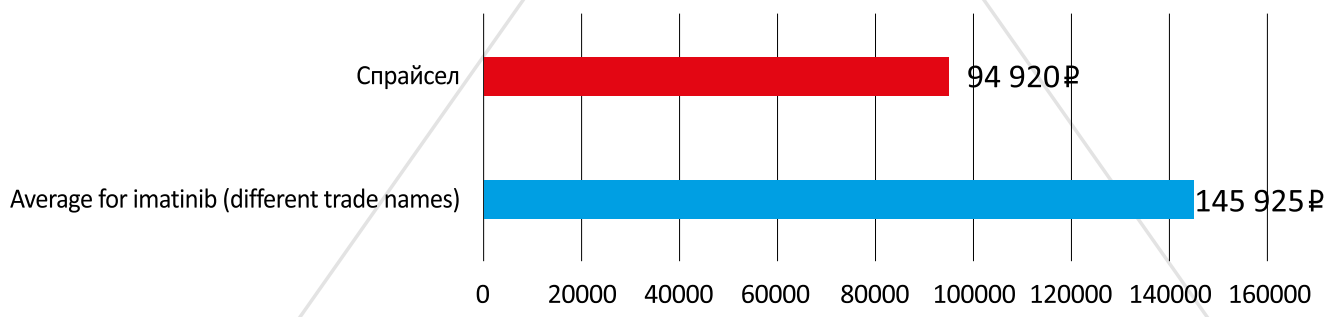

Figure 2. "Cost-effectiveness» analysis results

Scenario 1

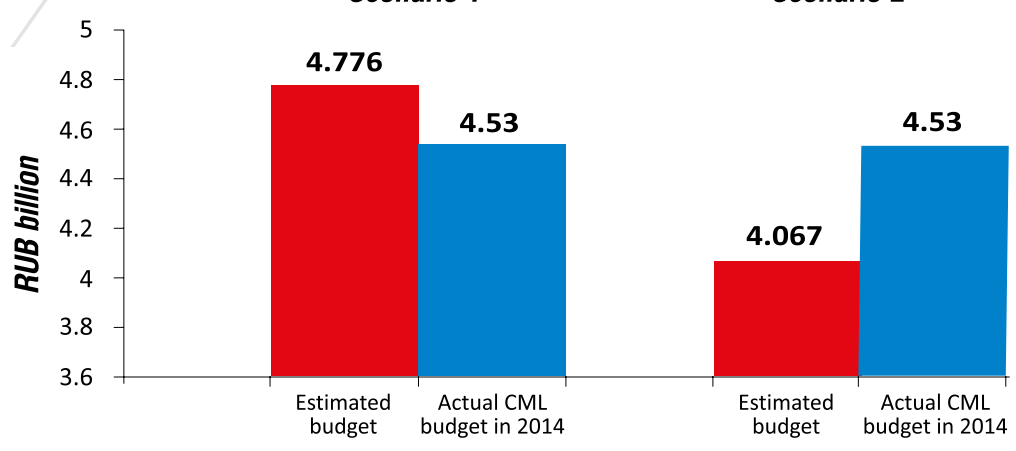

Figure 3. . Estimated budget when dasatinib is administered to $100 \%$ of imatinib-resistant patients versus the actual total budget for CML treatment in Russia in 2014 . For the purpose of modeling, an assumption was made regarding patients' adherence to dasatinib therapy based on a retrospective clinical study data (Scenario 1) or based on the analysis of the actual dasatinib consumption within the regional drug supply program in 2014 (Scenario 2). 


\section{Conclusion}

Described pharmacoeconomic analysis of dasatinib as a second-line therapy in CML patients with resistance and/or intolerance to imatinib demonstrated that:

- Dasatinib can be considered as a dominant second-line CML treatment alternative compared to high-dose imatinib according to the results of the cost-effectiveness analysis.

- With account for clinical study data on CML patients' treatment compliance rate, $82 \%$ of the imatinib-resistant and intolerant patients can be switched to a second-line therapy with dasatinib without additional funding compared to the actual total CML budget.

- If actual consumption rate of dasatinib supplied in frame of the regional budgets is considered, $100 \%$ of the imatinib-resistant and intolerant patients can be switched to dasatinib without total $\mathrm{CML}$ budget increase.

\section{References:}

1. National Clinical Guidelines on Chronic Myelogenous Leukemia Treatment and Diagnostics. A group of authors led by Academician V.G. Savchenko. 2014 2. Decree of the Government of the Russian Federation dated 02.10.2007 No. 1328-p On the List of Medicinal Products Procured Centrally from the Federal Budget

3. Decree of the Government of the Russian Federation dated 30.12.2014 No. 2782-p On the Approval of the Lists of Vital and Essential Medicinal Product for Human Use for 2015

4. Decree of the Government of the Russian Federation No. 871 dated August 30, 2014 On the Approval of the Rules for Drafting Lists of Medicinal Products for Human Use and the Minimal Range of Medicinal Products Required to Provide Medical Care

5. Kantarjian H, Pasquini R, Lévy V, et al. Dasatinib or high-dose imatinib for chronic-phase chronic myeloid leukemia resistant to imatinib at a dose of 400 to 600 milligrams daily: two-year follow-up of a randomized phase 2 study (START-R). Cancer. 2009 Sep 15;115(18):4136-47
6. Khabriev R.U., Kulikov A. Y., Arinina E.E. Methodological Fundamentals of Pharmacoeconomic Analysis. M. 2011.

7. Yagudina R.I., Serpik V.G., Sorokovikov I.V. Methodological Fundamentals of «Cost-Effectiveness" Analysis // Pharmacoeconomics: Theory and Practice. 2014. - Volume 2, No. 2. - Page 23-27

8. Yagudina R.I., Babiy V.V. Methodological Fundamentals of Effectiveness Analysis of Medical Technology in Pharmacoeconomic Studies // Pharmacoeconomics: Theory and Practice. - 2015. - Volume 3, No. 1. - Page 7-11

9. State Register of Medicinal Products [Electronic resource] - Accessible at: http://grls.rosminzdrav.ru. Date of access: 01.2015.

10. Electronic government procurement portal [Electronic resource] Accessible at: http://zakupki.gov.ru.

11. Esposti LD, Saragoni S, Buda S and Calandriello MI. Medication Adherence in Patients with Chronic Myelogenous Leukemia Using Tyrosine-Kinase Inhibitors_A Retrospective Analysis. Blood Vol 124 (21):1808-1808 December 6,2014

12. Experts in Chronic Myeloid Leukemia. The price of drugs for chronic myeloid leukemia (CML) is a reflection of the unsustainable prices of cancer drugs: from the perspective of a large group of CML experts // Blood. - May 2013, 121 (22) 4439-4442

13. Gorbacheva A. Chromosomal Fail // Nezavisimaya Gazeta. - 2015. - issue 03.03.2015

14. Jabbour E, Kantarjian H, O'Brien $S$ et al., The achievement of an early complete cytogenetic response is a major determinant for outcome in patients with early chronic phase chronic myeloid leukemia treated with tyrosine kinase inhibitors. Blood. 2011 Oct 27;118(17):4541-6

15. Neil P. Shah et al, Long-term outcome with dasatinib after imatinib failure in chronic-phasechronic myeloid leukemia: follow-up of a phase 3 study; Blood. 2014; Vol. 123, 15

16. Turkina A.G. et al. Russian Register of Chronic Myelogenous Leukemia Treatment in Routine Clinical Practice: Results of Many Years of Work // Effective Pharmacotherapy. - 10/2015

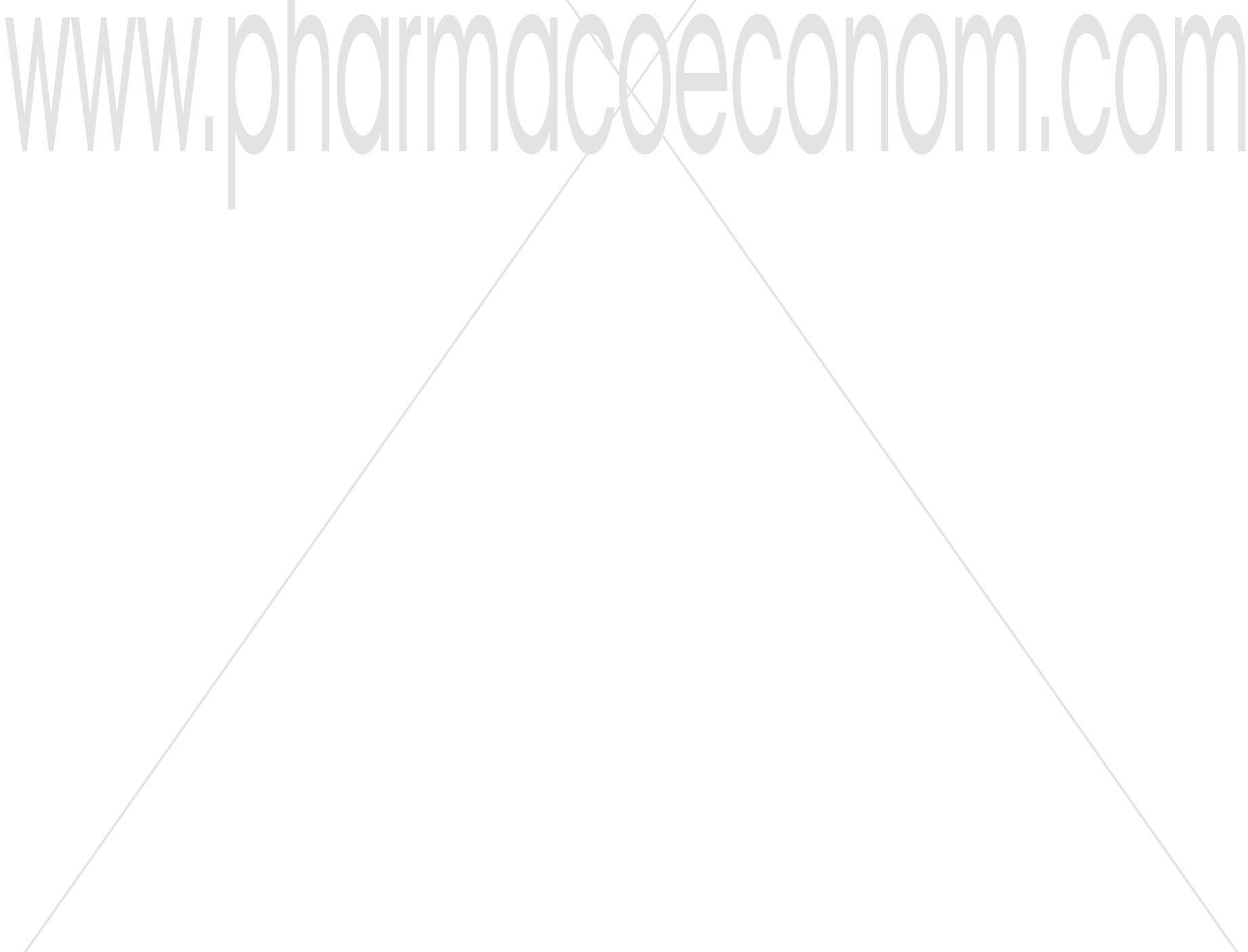

